\title{
POLINIZAÇÃO NATURAL E ARTIFICIAL DA CHERIMÓIA (Annona cherimola Mill.) NO ESTADO DE SÃO PAULO ${ }^{1}$
}

\author{
MARCELO ROSA MELO ${ }^{2}$, CELSO VALDEVINO POMMER ${ }^{3}$, RYOSUKE KAVATI ${ }^{4}$, TAKANOLI TOKUNAGA ${ }^{5}$
}

\begin{abstract}
RESUMO - Para estudar o método de polinização da cherimóia (Annona cherimola Mill.) que produza frutos em maior quantidade e melhor qualidade, instalou-se experimento em Pedra Bela - SP, a 1150 metros de altitude. As plantas eram de pé-franco, de 20 anos de idade. O experimento foi realizado em dois períodos, tendo o primeiro se iniciado em novembro de 1999 e finalizado em junho de 2000; o segundo iniciou-se em novembro de 2000 e encerrado em junho de 2001. A montagem do experimento foi efetuada no delineamento de blocos ao acaso, com 3 tratamentos e 12 repetições. Os tratamentos foram os seguintes: 1) polinização natural; 2) polinização manual cruzada; 3) autopolinização (flores ensacadas). As plantas dos blocos foram polinizadas em diferentes dias. Avaliaram-se o vingamento dos frutos 10 dias após a polinização e a quantidade dos frutos com conformação perfeita ou defeituosa aos 40 dias. Após a colheita, os frutos foram pesados individualmente. Foram também amostrados dois frutos de cada bloco (em 1999) e três (em 2000), para as seguintes determinações: massa das sementes e da polpa, número de sementes por 100 gramas de polpa. A polinização artificial proporcionou maior vingamento de frutos $(61,2 \%$ e 50,4\%) em relação à polinização natural $(19,6 \%$ e $3,3 \%)$, nos anos de 1999 e 2000 , respectivamente. Foi mais efetiva quando realizada sob condições de temperatura variando de $17^{\circ} \mathrm{C}$ até $22^{\circ} \mathrm{C}$ e umidade relativa do ar entre 70 e $80 \%$. Verificou-se também aumento da porcentagem de frutos perfeitos (em 2000), da massa do fruto e do índice de sementes/100 g de polpa dos frutos polinizados artificialmente em relação aos polinizados naturalmente.
\end{abstract}

Termos para indexação: Annona cherimola Mill., polinização artificial, polinização natural, anonácea, qualidade de fruto.

\section{NATURAL AND ARTIFICIAL POLLINATION OF CHERIMOYA (Annona cherimola Mill.) IN SÃO PAULO STATE}

\begin{abstract}
Experiments were carried out to determine which pollination method produces fruits of cherimoya (Annona cherimola Mill.) in larger amount and quality. The experiment was installed in a commercial orchard located in the county of Pedra Bela - SP, at an altitude of 1150 meters. The plants were in their own roots with an age of 20 years. The experiment was accomplished in two periods, the first began in November 1999 and concluded in June 2000; the second began in November of 2000 and was concluded in June 2001. The experimental design was in randomized blocks, with 3 treatments and 12 replicates. The treatments were the following ones: 1) natural pollination; 2) hand pollination (with mixture of pollen from different plants); 3) self-pollination (sacked flowers). The plants in the blocks were pollinated in different days. The evaluation of fruit set happened 10 days after the pollination and the amount of fruits with perfect or defective shape after 40 days. After harvest, the fruits were individually weighed. two fruit in 1999 and three fruit in 2000 of each treatment were taken, for the following determinations: seed and pulp weigh, number of seeds per 100 $\mathrm{g}$ of pulp. Hand pollination of cherimoya provided a larger fruit set $(61.2 \%$ and $50.4 \%)$ in relation to natural pollination (19.6\% and 3.3\%) in the years of 1999 and 2000, respectively. It was more effective when accomplished under temperature conditions varying from $17^{\circ} \mathrm{C}$ to $22^{\circ} \mathrm{C}$ and relative air humidity among 70 and $80 \%$. It was also verified an increase on the percentage of perfect fruits (in 2000), mass of the fruit and on the index of seeds/ $100 \mathrm{~g}$ of pulp of the fruits pollinated artificially in relation to the naturally pollinated.
\end{abstract}

Index terms: Annona cherimola Mill., hand pollination, natural pollination, fruit quality.

\section{INTRODUÇÃO}

Escolheu-se estudar a cherimóia, por ser fruta muito saborosa e que apresenta boas potencialidades econômicas de cultivo no Brasil (especialmente para pequenos produtores). Estima-se que sejam cultivados 10.000 ha de anonáceas no Brasil (Carmo, 2000), sendo que 1000 ha correspondem à atemóia, os quais são distribuídos em aproximadamente 500 ha na região Nordeste do País e 500 ha no Sudeste brasileiro. A cultura da cherimóia atualmente ocupa uma área de 25 ha no Estado de São Paulo, principalmente na região da Serra da Mantiqueira e de Itapetininga (Kavati, 1998). A cultura de atemóia, que é o híbrido entre Annona squamosa L. (fruta-do-conde ou ata) e Annona cherimola Mill. (cherimóia), proporciona boa remuneração ao produtor, pois uma planta adulta, manejada adequadamente, pode produzir até 20 caixas de frutos de boa qualidade (Tokunaga, 2000).

As flores de cherimóia possuem três pétalas carnosas de 4,0 $\mathrm{cm}$, são hermafroditas, aromáticas e pouco chamativas. A parte masculina da flor é constituída de 200 estames, dispostos helicoidalmente. A parte feminina é composta de 150 carpelos, dispostos em espiral, formando um cone compacto. As flores apresentam o fenômeno de dicogamia protogínica, que é a falta de sincronia no amadurecimento dos órgãos florais feminino e masculino dessas fruteiras. O estádio feminino inicia-se às $13 \mathrm{~h}$ e dura aproximadamente 26 horas e caracterizase pela receptividade dos estigmas. O estádio macho inicia-se após o estádio fêmea e dura 2 horas, e caracteriza-se pela liberação do pólen, com o estigma tornando-se não-receptivo.

A produtividade da cherimóia, porém, é baixa, devido à inexistência de cultivares adaptadas às diferentes condições regionais, escassez de informações a respeito dos tratos culturais, além da polinização natural deficiente, devido à ocorrência da dicogamia protogínica.

Para incrementar a produtividade, Schroeder, citado por Richardson \& Anderson (1996), propôs, para as condições da Califórnia - EUA, o emprego da polinização artificial ou manual. A polinização artificial, por sua vez, apresenta a vantagem de produzir frutos em maiores quantidades, de maior tamanho e de melhor forma. Com seu uso, pode-se aproveitar melhor a primeira florada, escalonar a colheita dos frutos e manejar a colheita, polinizando as flores das partes mais baixas da planta (Guirado, 1992). Por outro lado, esta operação eleva os custos de produção da cultura, pois exige 200 horas de trabalho para polinizar um hectare, visando a obter produtividade de 10 t/ha (Farré \& Hermoso, 1997).

O presente trabalho visou a comparar distintas maneiras de polinizar a cherimóia nas condições climáticas paulistas e a verificar os efeitos da polinização na quantidade e qualidade dos frutos produzidos.

\section{MATERIALEMÉTODOS}

1 (Trabalho 171/2001). Recebido: 18/10/2001. Aceito para publicação: 10/10/2002

2 Parte da dissertação de mestrado do primeiro autor apresentada ao Curso de Pós-Graduação em Agricultura Tropical e Subtropical do IAC.

3 Eng. ${ }^{\circ}$ Agr., Mestre em Agricultura Tropical e Subtropical/IAC. Bolsista da FAPESP. Tel. (11) 6979-3714. E-mail: rosamelo1 @ yahoo.com

4 Pesquisador Científico do Centro APTA Frutas do IAC, CP 28, 13001-970, Campinas. Bolsista do CNPq. (19) 3241-9910. E-mail: pommer@iac.br

5 Eng. ${ }^{\circ}$ Agrônomo MS, Coordenadoria de Assistência Técnica Integral. Tel. (14) 522-1388. E-mail: catilins @linsnet.com.br

6 Eng. ${ }^{\circ}$ Agrônomo, Coordenadoria de Assistência Técnica Integral. tel.: (11) 4582-7284 
O experimento de polinização em cherimóias foi realizado em pomar comercial, em Pedra Bela, distante $30 \mathrm{~km}$ de Bragança Paulista, situada a 1150 metros acima do nível do mar. O pomar atualmente é composto por 150 plantas de 20 anos de idade, propagadas a partir de sementes obtidas na própria região. O experimento ocorreu no período de novembro de 1999 a abril de 2000, sendo repetido no período de novembro de 2000 a maio de 2001. No final de cada período, os frutos foram colhidos e avaliados.

$\mathrm{O}$ delineamento experimental empregado foi o de blocos ao acaso (DBC), com 3 tratamentos e 12 repetições, cujos tratamentos foram os seguintes: 1) Polinização natural; 2) Polinização manual (mistura de pólen de outras plantas); 3) Autopolinização (incluído no ano 2000). Cada parcela foi constituída de 20 flores. Os três tratamentos foram aplicados em cada planta de cherimóia escolhida. A polinização foi iniciada às 6h30min e finalizada às $10 \mathrm{~h}$, nos dias 18-11, 26-11, 3-12-99; 27-1100, 3-12, 9-12 e 22-12-00. O referido horário foi determinado com a intenção de evitar o ressecamento do estigma que poderia ocorrer após as 10 horas, nos dias quentes e secos.

Foram escolhidas 12 plantas de mesma idade e vigor. Foram empregadas vinte flores por tratamento em cada bloco (planta). As flores dos tratamentos 2 e 3 foram protegidas quando estivessem prestes a abrir-se (estádio pré-fêmea), com saquinhos confeccionados com tecido chamado voil, que é usado para confeccionar véus. Os saquinhos foram abertos no instante da polinização e imediatamente fechados para assegurar que não haveria a interferência de agentes polinizadores externos. Os saquinhos foram retirados no momento da primeira avaliação, no décimo dia após a polinização.

$\mathrm{Na}$ área experimental, foi instalado um miniposto meteorológico para obter-se dados sobre temperatura e umidade relativa do ar.

Para obter o pólen, foi adotada a metodologia, adaptada de Guirado (1992), que consistiu do seguinte: A partir das 15 h, foi colhido aleatoriamente, número de flores igual ao número que seria polinizado na manhã seguinte, as quais se encontravam em antese, no estádio fêmea e que ainda não tivessem passado para o estádio macho. Levadas ao galpão, as flores foram distribuídas em camada única nos pratos plásticos, para evitar fermentação do pólen e mantidas em condições ambientais naturais. Pela manhã seguinte, às $6 \mathrm{~h}$, as flores nos pratos foram transportadas para o campo e mantidas em caixa de papelão com tampa. Conforme a necessidade do pólen, ele era extraído das flores com os dedos. A seguir, com o auxílio de um pincel, o pólen era agrupado e colocado no aplicador de pólen. No segundo ano do experimento, as flores a serem tratadas foram ensacadas um dia antes, o que não ocorreu no primeiro ano.

Avaliou-se a porcentagem de vingamento de frutos 10 dias após a polinização; a porcentagem de frutos perfeitos, quarenta dias após a polinização. Os frutos colhidos foram pesados logo após a colheita, com o auxílio de uma balança com precisão de 0,1 grama, de marca Marte. Para as avaliações a seguir, foram amostrados, sempre que possível, dois frutos por planta no ano de 1999 e três no ano de 2000: massa de polpa, massa das sementes e número de sementes por 100 gramas de polpa.

\section{RESULTADOS E DISCUSSÃO}

Observa-se, nos anos estudados, que o número médio de frutos vingados foi maior para o tratamento 2 (polinização artificial) seguido do tratamento 1 (polinização natural) e do tratamento 3 (autopolinização), resultados estatisticamente significativos, conforme se vê na Tabela 1. Rubi et al. (1997), no México, trabalhando com cherimóia de pé-franco, obtiveram 50,0\% de vingamento com polinização artificial.

Rubi et al. (1997) obtiveram valores de $0,8 \%$ para o vingamento natural em seus experimentos de polinização, similares aos valores das porcentagens de frutos vingados naturalmente aqui encontrados no ano de 2000. Entretanto, em 1999, os valores de vingamento natural foram maiores do que os obtidos no tratamento de polinização natural em 2000 e bastante superiores aos daqueles autores.
No ano de 2000, o vingamento natural diminuiu em relação a 1999, mas este valor foi semelhante aos observados por Caldeira et al. (1995), que foram de 4,8 \% de vingamento natural, para a média das cultivares Madeira, Mateus I e Reconversão, polinizadas na ilha da Madeira. Esses autores observaram que a porcentagem de vingamento da polinização natural oscilou ao longo do florescimento, variando de $0,0 \%$ a $24,0 \%$ para a cultivar Mateus I. Esse maior vingamento ocorreu em condições de temperaturas mais amenas.

Para o vingamento dos frutos, a umidade relativa do ar (UR) influencia os resultados, pois, no ano de 1999, a maior porcentagem de vingamento foi obtida para a polinização realizada no dia 26-11 (Tabela 2), quando a UR era de 70 a $80 \%$ e a temperatura entre $17^{\circ} \mathrm{Ce} 22^{\circ} \mathrm{C}$ (Tabela 3). O vingamento foi menor para polinizações feitas em 18-11 e 3-12. Nesses dias, as oscilações de temperatura e umidade no período de polinização, respectivamente, foram $12,3^{\circ} \mathrm{C}$ a $22,8^{\circ} \mathrm{C}$ e $97 \%$ a $61 \%$ e 15,5 ${ }^{\circ} \mathrm{Ca} 23,1^{\circ} \mathrm{Ce} 93 \%$ a $62 \%$ (Tabela 3 ).

Diante do que foi observado no experimento, é possível deduzir que esta baixa porcentagem de vingamento pode ser compensada polinizando-se mais flores.

TABELA 1 - Número médio de frutos de cherimóia vingados por planta e porcentagem obtidos em ensaios de polinização. Pedra Bela, 1999 e 2000.

\begin{tabular}{|c|c|c|c|c|}
\hline \multirow{2}{*}{ Tratamentos } & \multicolumn{2}{|c|}{$\mathrm{N}^{\circ}$ médio de frutos vingados por planta } & \multicolumn{2}{|c|}{ Porcentagem de frutos vingados } \\
\hline & 1999 & 2000 & 1999 & 2000 \\
\hline 1 & $3,9 \mathrm{~b}$ & $0,7 \quad b$ & $19,6 \quad b$ & 3,3 \\
\hline 2 & $12,5 \mathrm{a}$ & $10,1 \mathrm{a}$ & $61,2 \mathrm{a}$ & $50,4 \mathrm{a}$ \\
\hline 3 & - & $0,2 \mathrm{~b}$ & - & 0,2 \\
\hline $\mathrm{F}$ (trat) & $164,9^{*}$ & $41,6^{*}$ & $34,9 *$ & $41,6^{*}$ \\
\hline $\mathrm{CV}(\%)$ & 71,4 & 153,4 & 71,4 & 153,4 \\
\hline
\end{tabular}

Tratamentos: 1) Polinização natural; 2) Polinização artificial; 3) Autopolinização. Médias seguidas das mesmas letras nas colunas não diferem entre si, pelo teste de Tukey, a $5 \%$.*Significativo a $5 \%$.

TABELA 2 - Porcentagem de frutos vingados de cherimóia por polinização natural e artificial em diferentes datas de polinização. Pedra Bela, 1999.

\begin{tabular}{ccccc}
\hline Tratamentos & $18-11-1999$ & $26-11-1999$ & $3-12-1999$ & Médias \\
\hline 1 & $29,2 \mathrm{~b}$ & $3,3 \mathrm{a}$ & $16,7 \mathrm{a}$ & $19,6 \quad \mathrm{~b}$ \\
2 & $65,0 \mathrm{a}$ & $76,7 \mathrm{a}$ & $38,3 \mathrm{a}$ & $62,5 \mathrm{a}$ \\
\hline F (trat) & $21,7^{*}$ & $1,67^{\mathrm{ns}}$ & $10,57^{\mathrm{ns}}$ & $34,9^{*}$ \\
CV (\%) & 42,7 & 100,9 & 75,2 & 70,3 \\
\hline
\end{tabular}

Tratamentos: 1) Polinização natural; 2) Polinização artificial. Médias seguidas das mesmas letras nas colunas não diferem entre si, pelo teste de Tukey, a $5 \%$. Significativo a $5 \%$.

TABELA 3 - Temperatura e umidade relativa do ar observadas durante a polinização de cherimóias. Pedra Bela-SP, 1999.

\begin{tabular}{ccccccccccc}
\hline & \multicolumn{10}{c}{ Horário } \\
\cline { 2 - 11 } Datas & \multicolumn{1}{c}{7} & \multicolumn{1}{c}{8} & \multicolumn{2}{c}{9} & \multicolumn{2}{c}{10} & \multicolumn{2}{c}{11} \\
\cline { 2 - 11 } & $\mathrm{T}^{0} \mathrm{C}$ & UR $\%$ & $\mathrm{~T}^{0} \mathrm{C}$ & $\mathrm{UR} \%$ & $\mathrm{~T}^{0} \mathrm{C}$ & $\mathrm{UR} \%$ & $\mathrm{~T}^{0} \mathrm{C}$ & $\mathrm{UR} \%$ & $\mathrm{~T}^{0} \mathrm{C}$ & $\mathrm{UR} \%$ \\
\hline $18-11$ & 12,3 & 97 & - & - & - & - & 19,0 & 72 & 22,8 & 61 \\
$26-11$ & 17,0 & 76 & 22,0 & 70 & 18,5 & 80 & - & - & - & - \\
$3-12$ & 15,5 & 93 & - & - & 23,1 & 62 & - & - & - & - \\
\hline
\end{tabular}

No ano 2000, o melhor resultado foi alcançado no dia 27-11 (Tabela 4), quando a UR variou de $100 \%$ a $67 \%$ e a temperatura entre $17,5^{\circ} \mathrm{Ce} 25^{\circ} \mathrm{C}$ (Tabela 5). $\mathrm{O}$ vingamento foi menor para polinizações realizadas nos dias 3-12, 9-12 e 22-12 (Tabela 4). Nessas datas, as oscilações de temperatura foram, respectivamente: $17,5^{\circ} \mathrm{C}$ e $27,5^{\circ} \mathrm{C}$ e $100 \%$ a $57 \% ; 17,5^{\circ} \mathrm{C}$ e $28,2^{\circ} \mathrm{C}$ e $98 \%$ a $53 \%$ e finalmente $18^{\circ} \mathrm{C}$ e $26,9^{\circ} \mathrm{C}$ e $99 \%$ a $65 \%$ (Tabela 5). 
TABELA 4 - Porcentagem de frutos vingados de cherimóia por polinização natural e artificial em diferentes datas de polinização. Pedra Bela, 2000

\begin{tabular}{crrrrr}
\hline Tratamentos & \multicolumn{3}{c}{ Datas } & Médias \\
\cline { 2 - 6 } & $27-11-2000$ & $3-12-2000$ & $9-12-2000$ & $22-12-2000$ \\
\hline 1 & $8,3 \mathrm{~b}$ & $1,2 \mathrm{~b}$ & $0,0 \mathrm{a}$ & $3,3 \mathrm{~b}$ & 3,3 \\
2 & $73,3 \mathrm{a}$ & $36,2 \mathrm{a}$ & $25,0 \mathrm{a}$ & $63,3 \mathrm{a}$ & 50,4 \\
3 & $0,0 \mathrm{~b}$ & $1,2 \mathrm{~b}$ & $2,5 \mathrm{a}$ & $0,0 \mathrm{~b}$ & 0,8 \\
\hline $\mathrm{F}$ (trat) & $19,13^{*}$ & $8,40^{*}$ & $7,00^{\text {ns }}$ & $195,9^{*}$ & 41,6 \\
CV (\%) & 140,8 & 167,0 & 151,9 & 139,6 & 153,2 \\
\hline
\end{tabular}

Tratamentos: 1) Polinização natural; 2) Polinização artificial; 3) Autopolinização. Médias seguidas das mesmas letras nas colunas não diferem entre si, pelo teste Tukey, a $5 \%$ * Significativo a $5 \%$.

TABELA 5 - Temperatura e umidade relativa do ar observadas durante a polinização de cherimóias. Pedra Bela-SP, 2000

\begin{tabular}{|c|c|c|c|c|c|c|c|c|c|c|c|c|}
\hline \multirow{4}{*}{ Datas } & \multicolumn{12}{|c|}{ Horário } \\
\hline & \multicolumn{2}{|c|}{7} & \multicolumn{2}{|c|}{8} & \multicolumn{2}{|c|}{9} & \multicolumn{2}{|c|}{10} & \multicolumn{2}{|c|}{11} & \multicolumn{2}{|c|}{12} \\
\hline & $\mathrm{T}$ & UR & $T$ & UR & $\mathrm{T}$ & UR & $\mathrm{T}$ & UR & $\mathrm{T}$ & UR & $T$ & UR \\
\hline & ${ }^{0} \mathrm{C}$ & $\%$ & ${ }^{0} \mathrm{C}$ & $\%$ & ${ }^{\circ} \mathrm{C}$ & $\%$ & ${ }^{0} \mathrm{C}$ & $\%$ & ${ }^{0} \mathrm{C}$ & $\%$ & ${ }^{0} \mathrm{C}$ & $\%$ \\
\hline 27-11 & 17,5 & 100 & 18,5 & 100 & 22,5 & 90 & 24,0 & 75 & 24,5 & 72 & 25,0 & 67 \\
\hline 03-12 & 17,5 & 100 & 20,5 & 98 & 23,5 & 75 & 24,7 & 61 & 26,2 & 57 & 27,5 & 58 \\
\hline 09-12 & 17,5 & 98 & 21,0 & 83 & 25,0 & 72 & 26,3 & 62 & 27,2 & 58 & 28,2 & 53 \\
\hline $22-12$ & 18,2 & 99 & 21,0 & 88 & 25,0 & 69 & 25,2 & 67 & 26,4 & 67 & 26,9 & 65 \\
\hline
\end{tabular}

Os tratamentos não influenciaram a porcentagem de frutos perfeitos em 1999 (Tabela 6). Os tratamentos apresentaram diferenças significativas em termos de porcentagem de frutos perfeitos no ano de 2000, sendo o tratamento de polinização artificial superior aos demais, reforçando a importância desta prática cultural no incremento da renda do produtor rural, ao produzir maior quantidade de frutos comercializáveis.

Os tratamentos 1 e 2 mostraram diferenças significativas para massa dos frutos (Tabela 7). O tratamento 2 produziu frutos de maior tamanho em 1999 e 2000.

TABELA 6 - Porcentagem de frutos perfeitos

\begin{tabular}{|c|c|c|}
\hline \multirow{3}{*}{$\begin{array}{c}\text { Tratamentos } \\
1\end{array}$} & \multicolumn{2}{|c|}{ Porcentagem de frutos perfeitos } \\
\hline & 1999 & 2000 \\
\hline & 35,4 a & $18,7 \quad b$ \\
\hline 2 & $64,0 \quad a$ & 72,8 a \\
\hline 3 & - & $8,3 \quad b$ \\
\hline F (trat) & ns & $19,1 *$ \\
\hline $\operatorname{CV}(\%)$ & 89,2 & 127,3 \\
\hline
\end{tabular}

Tratamentos: 1) Polinização natural; 2) Polinização artificial; 3) Autopolinização. Médias seguidas das mesmas letras nas colunas não diferem entre si, pelo teste Tukey, a 5\%. *Significativo a 5\%.

TABELA 7 - Influência dos tratamentos sobre alguns atributos dos frutos de cherimóia. Pedra Bela, 1999 e 2000.

\begin{tabular}{|c|c|c|c|c|c|c|c|c|c|c|c|c|c|c|c|c|c|c|}
\hline & \multicolumn{2}{|c|}{$\begin{array}{l}\text { Massa dos frutos } \\
\text { (g) }\end{array}$} & \multicolumn{4}{|c|}{$\begin{array}{l}\text { Número de sementes } \\
\text { (un) }\end{array}$} & \multicolumn{4}{|c|}{$\begin{array}{l}\text { Massa de sementes } \\
\text { (g) }\end{array}$} & \multicolumn{4}{|c|}{$\begin{array}{l}\text { Massa de polpa } \\
(\mathrm{g})\end{array}$} & \multicolumn{4}{|c|}{$\begin{array}{l}\text { Número de sementes/100 } \\
\text { g de polpa }\end{array}$} \\
\hline Trat. & 1999 & 2000 & & 999 & 20 & & 19 & 99 & 200 & & 199 & 999 & 200 & & & 1999 & & 000 \\
\hline 1 & $126,5 b$ & $155,0 \mathrm{~b}$ & & 21,5 & 17 & 1 & & 3,8 & 9,9 & & & 1,0 & 240, & & & 9,2 & & 6,6 \\
\hline 2 & $407,7 \mathrm{a}$ & $563,3 \mathrm{a}$ & & 29,5 & 40 & 1,7 & & 7,6 & 20, & & & 1,0 & 443, & & & 11,1 & & 9,3 \\
\hline \multirow[t]{2}{*}{3} & - & $44,2 b$ & & - & 14 & 0 & & - & 7,7 & & & - & 198, & & & - & & 7,0 \\
\hline & & & & & Inter & valo & & de & confia & & & & & & & & & \\
\hline 1 & - & - & 12,7 & 30,3 & 4,0 & 30,2 & 7,9 & 19,7 & 2,2 & 17,6 & 180 & 302 & 172 & 409 & 5,5 & 13,0 & 5,5 & 7,7 \\
\hline 2 & - & - & 19,7 & 39,3 & 33,2 & 48,2 & 12,0 & 18,6 & 17,2 & 24,6 & 205 & 377 & 389 & 498 & 7,1 & 15,1 & 7,9 & 10,7 \\
\hline 3 & - & - & & - & & & & - & . & & & - & - & & & - & & - \\
\hline
\end{tabular}

Tratamentos: 1) Polinização natural; 2) Polinização artificial; 3) Autopolinização Médias seguidas das mesmas letras nas colunas não diferem entre si, pelo teste Tukey, a $5 \%$.
Nota-se que o número de sementes não foi diferente no ano de 1999, mas diferiu significativamente entre os tratamentos 1 e 2 em 2000, apresentando o tratamento 2 (polinização artificial) um número de sementes significativamente superior ao tratamento 1 . Isso demonstra, conforme Duarte e Escobar (1997) já haviam constatado anteriormente, que a polinização artificial fecundou maior quantidade de estigmas, formando maior número de sementes, que, por sua vez, se traduziram em frutos de maior tamanho. Com relação à autopolinização, não foi possível analisar os dados, devido ao pequeno número de frutos obtidos (dois).

Os intervalos de confiança da massa de sementes e massa de polpa demonstraram que não houve diferenças significativas em $1999 \mathrm{e}$ 2000 (Tabela 7).

Verificou-se, em 1999, que os tratamentos não influenciaram o número de sementes por 100 gramas de polpa, mas o tratamento 2 apresentou índice de sementes superior a 10 sementes por 100 gramas de polpa, que é uma característica negativa, conforme Scheldeman e Van Damme (1999). No ano seguinte, para o tratamento 2 , o índice número de sementes por fruto por $100 \mathrm{~g}$ de polpa foi maior e estatisticamente significativo, demonstrando que a polinização artificial aumentou o número de sementes. Como também ocorreu o aumento da massa de polpa (Tabela 7), o índice de sementes foi mantido abaixo do limite considerado indesejável.

\section{CONCLUSÕES}

1. A polinização artificial foi mais efetiva quando realizada em condições de temperatura de $17^{\circ} \mathrm{C}$ até $22^{\circ} \mathrm{C}$ e umidade relativa do ar entre 70 e $80 \%$. 2. A polinização artificial aumentou o vingamento dos frutos e, conseqüentemente, a produção.

3. A polinização artificial aumentou a porcentagem de frutos perfeitos em 2000, a massa do fruto e da polpa e o índice de sementes/100 g de polpa.

\section{REFERÊNCIASBIBLIOGRÁFICAS}

CALDEIRA, J.J.F.; ARAÚJO, J.A.; NUNES, R. Estudo da polinização natural e da polinização artificial na anoneira crescimento dos frutos. Revista de Ciências Agrárias, v.18, n.1, p.91-104, 1995.

CARMO, A.J. do. Atemóia vão bem no Noroeste de SP. Estado de São Paulo, São Paulo, 10 de maio de 2000. Suplemento Agrícola, n.2322, p.10-11, 2000.

DUARTE, O.; ESCOBAR, O. Mejora del cuajado de chirimoya (Annona cherimola Mill.) cv. Cumbe, mediante polinización manual autogama $\mathrm{y}$ alogama. Proceedings of the Interamerican Society for Tropical Horticulture, n.41, 162-165, 1997.

FARRE, J.M.; HERMOSO, J.M. El chirimoyo (Annona cherimola Mill.) en España. In: SÃO JOSÉ, A.R.; SOUZA, I.V.B.; MORAIS, O.M.; REBOUÇAS, T.N.H.(Ed.). Anonáceas, produção e mercado (Pinha, Graviola, Atemóia e Cherimólia). Vitória da Conquista: DFZ/UESB, 1997, p.84-87.

GUIRADO, E. Polinización artificial del chirimoyo. Caja Rural de Granada, 1992, 15p.

KAVATI, R. Efeito de épocas de poda na produção tardia e fenologia de atemóia (Annona cherimola Mill. X Annona squamosa L.), cv. Gefner 1998. 120f. Dissertação (Mestrado em Agronomia) Faculdade de Ciências Agrárias e Veterinárias, Universidade Estadual Paulista, Jaboticabal.

RICHARDSON, A.C.; ANDERSON, P.A. Hand pollination effects on the set and development of cherimoya (Annona cherimola Mill.) fruit in a humid climate. Scientia Horticulturae, v.65, p.273-281, 1996.

RUBI, M.A.; MARTINEZ, A.M.; LÓPEZ, L.L. Polinización manual en chirimoya y su relación con producción y características del fruto. In: CONGRESO INTERNACIONAL DE ANONÁCEAS, 1, 1997, Chapingo, México. Memorias... Chapingo: Universidad Autónoma de Chapingo, 1997. p.19-27.

SCHELDEMAN, X.; VAN DAMME, P. Accesiones promisorias de chirimoya en la provincia de Loja, sur del Ecuador. Acta horticulturae, Amsterdam, n.497, p.181-188, 1999. 